\title{
BARRERAS A LA PARTICIPACIÓN POLÍTICA DE LAS MUJERES CON DOBLE JORNADA LABORAL EN NUEVO LEÓN, MÉXICO
}

\section{Barriers to the political participation of women with double working hours in Nuevo Leon, Mexico}

\author{
Selene de la Fuente Rivera \\ Profesora investigadora de la Facultad de Ciencias \\ Políticas y Relaciones Internacionales de la Univer- \\ sidad Autónoma de Nuevo León (UANL). México. \\ ORCID: 0000-0001-5363-1381 \\ Correo-e: sdelafuenter@uanl.edu.mx \\ José Segoviano Hernández \\ Profesor investigador (UANL). \\ ORCID: 0000-0001-5236-2386 \\ Correo-e: jose.segovianhrz@uanl.mx
}

Xóchitl A. Arango Morales

Profesora investigadora (UANL).

ORCID: 0000-0002-0907-452X

Correo-e: xochitl.arangomr@uanl.edu.mx

Recibido: 9/2/2021 • Aprobado: 18/3/2021

Cómo citar: de la Fuente Rivera, S., Hernández, J. S., \& Arango Morales, X. A. (2021). Barreras a la participación política de las mujeres con doble jornada laboral en Nuevo León, México. Ciencia y Sociedad, 46(3), 59-75. Doi: https://doi.org/10.22206/cys.2021.v46i3. pp59-75

\section{Resumen}

En la presente investigación se abordan los resultados de un estudio dirigido a identificar las principales barreras de participación política que enfrentan las nuevoleonesas con doble jornada laboral. Las participantes entrevistadas se conjuntaron a partir de tres grupos: A) Servidoras Públicas, B) Integrantes de Organizaciones No Gubernamentales (ONG) y C) Grupos de la Sociedad Civil Organizada (GSCO). El estudio se estableció desde una perspectiva cualitativa, se recabaron las experiencias de las participantes mediante entrevistas en profundidad semiestructuradas y el análisis de la información fue por medio del software NVivo 11. En un primer momento se analizaron los discursos de las entrevistadas y en un segundo análisis se identificaron las barreras a las que se enfrentan en el ejercicio de la política. Los hallazgos encontrados se analizaron en cada uno de los tres grupos y se identificó que las barreras para participar en política son diferenciadas entre ellas, además que responden a condiciones y estructuras sociales como: entornos de clase social, familiar y de género. Las principales barreras identificadas

\begin{abstract}
This research addresses the results of a study aimed at identifying the main barriers to conventional and unconventional political participation, faced by women from Nuevo León with doble working hours. The interviewed participants came together from three groups: A) Public Servants, B) Members of Non-Governmental Organizations (NGOs), and C) Organized Civil Society Groups (GSCO). The study was established from a qualitative perspective, the experiences of the participants were collected through semi-structured in-depth interviews and the information analysis was through the Nvivo 11 software. At first, the speeches of the interviewees were analyzed and in a second analysis identified the barriers they face in the exercise of politics. The findings found were analyzed in each of the three groups and it was identified that the barriers to participating in politics are differentiated among them, in addition to responding to social conditions and structures such as: social class, family and gender environments. The main barriers identified were dedication to motherhood, electoral political inequity,
\end{abstract}


fueron: la dedicación a la maternidad, la inequidad política electoral, la violencia política en razón de género y la represión, la sociedad patriarcal, el problema de movilidad urbana, el límite para acceder al sistema de guarderías, además de la instauración de estereotipos de género que conducen al cuidado exclusivo de la familia.

Palabras clave: participación política, mujer en política, participación de la mujer, empleo de las mujeres.

\section{Introducción}

Las mujeres en el espacio público se han visto sujetas históricamente a una serie de estereotipos que se acompañan de hábitos y costumbres, que han facilitado retenerlas en el escenario del trabajo del hogar y el cuidado de la familia. No obstante, la participación de las mujeres en el aporte de la economía familiar las ha posicionado cada vez más como tomadoras de decisiones, incluyendo su presencia en la arena política.

En la actualidad, las mujeres en México han logrado incrementar los niveles de participación política electoral con la aplicación de la Ley de Paridad de Género del año 2014, sumando posiciones sustantivas en la participación política. Esta ley exige y obliga a los partidos políticos a garantizar la postulación de candidaturas para legislaturas federales y locales con equidad de género, es decir, integrar las candidaturas por $50 \%$ de mujeres y $50 \%$ de hombres.

Las modificaciones realizadas a la Ley General de Instituciones y Procedimientos Electorales (LEGIPE) y a la Ley General de Partidos Políticos (LGPP), sustituyen al anterior Código Federal de Instituciones y Procedimientos Electorales (COFIPE). Sin embargo, el sustento legal que sitúa en condiciones iguales tanto a hombres como a mujeres para participar en el espacio político-electoral no es suficiente para alcanzar las formas de participación política de las mujeres de manera natural, sin que se vean obligados los partidos políticos a cumplir con lo que la norma les requiere. political violence based on gender and repression, patriarchal society, the problem of urban mobility, limits to access the day care system, in addition to the establishment of gender stereotypes that lead to exclusive care of the family.

Keywords: Political participation, women in politics, women's participation, women's employment.

Las explicaciones de la baja visibilidad de la participación política de las mujeres pueden ser múltiples; sin embargo, diversos estudios (Benhabib, 1997; Butler, 2007; Peris Vidal, 2013) coinciden en que las mujeres han quedado fuera del ámbito político porque la política se relaciona íntimamente con el espacio público, mientras que el desarrollo práctico de las mujeres se concentra y se limita erróneamente al ámbito privado, pese a que su participación es cada vez mayor en el mercado laboral, es decir, fuera del hogar.

En este mismo sentido, los resultados del estudio de Vázquez, Cárcamo \& Hernández (2012) concluyen que cuando la mujer adquiere un puesto de prestigio en un trabajo remunerado no la exenta del trabajo en el hogar y de los cuidados de sus integrantes, ni tampoco crea cambios en la distribución equitativa de las tareas del hogar. De igual manera, la Asociación Latinoamericana de Organizaciones de Promoción al Desarrollo (ALOP) en su informe regional sobre la participación política de las mujeres, señala que la carga exclusiva para la mujer del trabajo en el hogar y de los cuidados, aunado a la jornada laboral remunerada, son elementos irreconciliables con la vida pública de las mujeres (Donoso \& Valdés, 2007).

Es por lo anterior que en el presente estudio se plantea el objetivo de identificar las barreras a las que se enfrentan las mujeres nuevoleonesas con doble jornada laboral en el ejercicio de la participación política. El abordaje del estudio es con un enfoque cualitativo que se propone conocer las experiencias de tres grupos de mujeres con presencia en el espacio de la política nuevoleonesa. 
Los criterios de elegibilidad de las participantes fueron a partir de incluir en el estudio las diversas formas de participación política de las nuevoleonesas: participación política electoral y representación política, participación política mediante organizaciones legalmente constituidas y participación colectiva no formal ni constituida con carácter de desobediencia civil.

La clasificación de las mujeres participantes se organizó en tres grupos: A) Servidoras Públicas; B) Integrantes de Organizaciones No Gubernamentales (ONG); C) Grupos de la Sociedad Civil Organizada (GSCO).

La información recabada se procesó en el software NVivo 11, el cual permitió generar un análisis general del discurso para cada grupo del estudio y se recogieron diferentes experiencias de las participantes entrevistadas. Con la interpretación de los datos analizados se logró identificar las barreras existentes en los grupos de mujeres estudiados, pudiendo resaltar la diferenciación de recursos lingüísticos propios de cada grupo y las barreras en cada uno de ellos.

\section{Contexto de la participación de la mujer al servicio de la sociedad: desde la esfera privada a la pública}

Las dinámicas de desarrollo que practican las personas, ya sea en el espacio privado o público, tienen relación con las condiciones materiales, de género e históricas en las cuales han vivido. Además de ello, hay un elemento que concierne directamente a la ubicación de las mujeres en el espacio privado, que se arraiga mediante el establecimiento de roles de género a partir del sexo biológico de las personas al nacer.

Los roles de género se transmiten de generación en generación y crean estereotipos acompañados de hábitos y costumbres. En este sentido, se ha utilizado el hogar como el escenario naturalizado para las mujeres y como destinataria de la responsabilidad del trabajo del hogar y los cuidados de quienes lo integran. Estos roles contienen una especie de expectativas consensuadas en la sociedad respecto a las acciones usuales pensadas para hombres y/o mujeres y que se vuelven normas prescriptivas para cada sexo (Godoy \& Mladinic, 2009).

Estos hábitos marcados por el género han desembocado en comportamientos que proyectan prejuicio para el desarrollo con equidad, igualdad y justicia entre hombres y mujeres. Desde la perspectiva del materialismo histórico, el desarrollo de las mujeres es paralelo a la desigualdad, con la ayuda de construcciones sociales asignadas al nacer [o desde antes], que justifican cualquier desarrollo desfavorecedor hacia las mujeres. Con la reproducción de patrones tradicionales se asignan roles basados en el género, que son encausados por la asimetría de poder y por la desigualdad, y que a su vez se sostienen porque representan el estilo de vivir de la sociedad, lo cual desencadena en la conformación de la cultura capitalista basada en la persecución del poder y el sometimiento de una clase o un grupo sobre otro (Fernández, 2007).

En este sentido, los aportes realizados desde la fuerza del trabajo de las mujeres que salen de los hogares contribuyen a mantener y mover al mundo (Federici, 2013). Estos trabajos tienden hacia acciones concretas: cuidados en salud física y psicoemocional, administración del hogar, de mantenimiento y limpieza, cuidado a menores, adultos mayores, condiciones adjudicadas hacia las mujeres, desde el desarrollo de las comunidades primitivas y a partir del desarrollo del capitalismo.

La responsabilidad de estos trabajos recae mayoritariamente en mujeres, pero además se le adjudican a las personas no ocupadas, la población con bajos ingresos y las personas con más edad (La Parra, 2001). Este mismo autor señala que este sistema de bienestar de los cuidados que se brindan en los 
hogares es realizado mayoritariamente por mujeres no ocupadas, con un bajo nivel de ingreso y una elevada edad.

Sin duda alguna los aportes que se trabajan desde el espacio privado, es decir, el hogar, han permitido el desarrollo de actividades en el espacio público de todas las personas que se beneficien de este trabajo. Se crean las condiciones para el desarrollo de las relaciones sociales y materiales que rodean la época contemporánea.

Federici (2013) hace una crítica a Marx respecto a que no hace una diferencia entre la producción de mercancías y la producción de la fuerza de trabajo, es decir, que el valor de la fuerza de trabajo se mide en función del valor de las mercancías como alimento, vivienda y vestido para asegurar su reproducción. También señala que es consecuencia del momento histórico que le toca narrar al citado autor, además de que, aún con la explicación histórica y política, sí reconoció el trabajo doméstico como una rama específica de la producción capitalista. Sin embargo, Federici es determinante a la hora de señalar que bajo la visión "tecnologisista" de la revolución, Marx ignoró el trabajo reproductivo que se desarrolla desde los hogares y por las mujeres, porque "compartía el mismo criterio que el capitalismo sobre qué constituye trabajo y qué no, y porque creía en el trabajo industrial asalariado como el estadio en el que se desarrollaría la batalla por la emancipación de la humanidad" (p. 175).

Desde este enfoque tradicional de la economía se ha generado una especie de invisibilidad hacia el trabajo del hogar, de los cuidados y de quién los realiza, que son mayoritariamente las mujeres. Además, con el auge del desarrollo capitalista lo significativo está dirigido a quienes producen y consumen, fuera de este binomio quedan las relaciones sociales subjetivas entre las personas, de las que resultan los cuidados de vida brindados por las mujeres hacia los miembros de las familias y por conclusión a la sociedad. Es por ello que la economía feminista brinda los principales aportes a la hora de medir el impacto económico del trabajo realizado desde los hogares.

Esta forma de comprender las amplias dimensiones en las que intervienen las actividades hechas desde el espacio privado permite identificar la relación del consumo de bienes y la provisión de fuerza de trabajo, porque garantizan la reproducción de sus miembros en sociedad. Rodríguez (2015) señala que podría decirse que el trabajo del hogar y de los cuidados no remunerados (y que realizan mayoritariamente las mujeres) constituye un subsidio a la tasa de ganancia y a la acumulación del capital (p. 11).

Es decir, el trabajo que se efectúa desde los hogares ha sido designado sin valor en términos económicos, dado que no produce bienes intercambiables, no obstante, sí reproduce los elementos necesarios para la vida y la producción en el espacio público. Bajo la perspectiva feminista que marca este estudio, el trabajo doméstico facilita el movimiento del capital, ya que este lo puede utilizar en función de sus necesidades, porque abastece fuerza de trabajo y porque conserva una reserva de capital humano para fines productivos (Dávila, 2018). Esto es, las contribuciones y facilidades del trabajo del hogar y de los cuidados repercute en el desenvolvimiento de las personas a las que va dirigido; sumado también a que el trabajo desde el hogar se realiza mayoritariamente en condiciones precarias y con alto nivel de feminización de las tareas referidas (Silva, 2020).

Las realidades adversas a las que refiere la feminización del trabajo del hogar radican en que las mujeres quedan al margen del espacio privado y se suma a una de las barreras centrales a la hora de enfrentarse a las dinámicas productivas y sociales del espacio público; llevando a cabo serios inconvenientes para organizar el tiempo de cuidadoras y trabajadoras del espacio público, sometiéndose a condiciones laborales precarias y duras realidades socioeconómicas (Dávila, 2018). 
Las responsabilidades, cuidados y tareas ejercidas en el hogar por parte de las mujeres sin percibir una remuneración, acortan las posibilidades de que la mujer pueda recrearse en otros ámbitos como el profesional.

La participación de las mujeres en el espacio público se ha dado con la perspectiva de responder a las demandas económicas y precarias de las condiciones de vida en el marco del neoliberalismo en México. Además de que su inserción en el mundo del trabajo a partir del desarrollo del capitalismo e impulsado por los mercados de producción se ha dado a la par de sostener también las responsabilidades del cuidado doméstico-familiar (Ortega, 2019) y un entorno de doble esfuerzo a la hora de participar en otros sectores como el laboral y el político.

Ocampo (2016) señala que el desarrollo del capitalismo contemporáneo gestó relaciones de dominación con un carácter patriarcal, racista y clasista, dentro de las cuales las mujeres se insertan en el mercado laboral, llevando a cabo una doble presencia; es decir, estar bajo la corresponsabilidad y conciliación de tiempos (Escoto, 2020), lo que conduce a una sobrecarga de trabajo que repercute en afectar la trayectoria laboral y la salud de las mujeres.

Al respecto, Federici (2013) explica el significado de una doble carga laboral para las mujeres, donde no solo se incrementa su explotación, sino que se continúa reproduciendo el rol de cuidadora en diferentes espacios. Donde sea que miremos podemos observar que los trabajos llevados a cabo por mujeres son meras extensiones de la labor de amas de casa (p. 39).

Incluso las posiciones alcanzadas por las mujeres en el espacio público no las exime del trabajo del hogar y de los cuidados dentro de la esfera privada, además de que las expone a desigualdades con tendencia sistemática, expresada mediante la violencia política, económica y salarial. Encontrando principalmente: el desempleo femenino entre los sectores de mujeres jóvenes de la población, la brecha salarial entre hombres y mujeres, la dificultad en el acceso a cargos jerárquicos, la dificultad para la autonomía económica y superación de la pobreza (Ibarra, 2018).

Podemos reconocer que la división sexual del trabajo es un obstáculo estructural [sistémico] que dificulta también el empoderamiento de las mujeres (Ibarra, 2018).

El progreso de la presencia de las mujeres en el espacio público se desarrolla sin que se realice un rompimiento a la dedicación del trabajo del hogar y de los cuidados. Adicionalmente, en la actualidad se han enfatizado las condiciones sistémicas de desigualdad, precariedad y reducción de las redes de seguridad social, que afectan significativamente al desarrollo integral de las mujeres.

De este modo, la acumulación a partir de la desposesión es la táctica generalizada, donde los que ganan son pocos a costa de las pérdidas de la mayoría y donde las desigualdades mencionadas son una historia cotidiana que padecen las mujeres como resultado de ser las principales responsables de sostener la vida diaria generacionalmente (Tapia Marchina, 2020).

\section{Participación de las mujeres en el espacio público y las desigualdades en el entorno de la política}

El desarrollo de las mujeres en el espacio público tiene un nexo central con la visión económica contemporánea, donde la precarización ha promovido que las mujeres salgan a emplearse en un entorno con desigualdades frente al de los hombres. Esta perspectiva las ha dejado en desventaja frente a los hombres, al incluirse valores sociales, culturales y generacionales que complejizan el desenvolvimiento de las mujeres en lo público y que contribuyen a la permanente presencia en el espacio privado del hogar, como en el apartado anterior se expone. 
Los bajos puestos ejecutivos, políticos y de toma de decisión se componen por la jerarquización laboral que responde a las relaciones de género, la poca flexibilidad en horarios para el desarrollo familiar y laboral, y también al machismo en la cultura, lo que recae en diversos problemas para las mujeres al no poder desempeñar su trabajo en el espacio público, quienes además tienen que vérselas por su cuenta con la doble jornada (Zabludovsky, 2007).

A lo largo de la historia y en cuanto a la representación y formas de participación política, los hombres han figurado primordialmente en el espacio público y político, creando el fenómeno de la masculinización de la política (Álvarez, 2011; Cerva, 2014). En consecuencia, ha gestado un estado de violencia política en contra de las mujeres, fenómeno que busca acortar las aspiraciones de involucrarse políticamente por el hecho de ser mujeres (Lena Krook \& Restrepo Sanín, 2016).

Las agresiones a las que las mujeres históricamente se han enfrentado tienen la condición de ser violencias simbólicas y materiales, dando espacio a la producción de violencia fusionada a estructuras marcadas de desigualdad, discriminación y opresión (Galarza Fernández, Cobo Bedía, \& Esquembre Cerdá, 2016).

La violencia generada contiene características que la hacen invisible para quienes no reflexionan desde un enfoque sistémico. Se manifiesta de forma gradual y por distintos medios, es acumulativa, difícil de atribuir a un agente en particular, e invisible incluso para sus propias víctimas (Pérez, 2019).

En este sentido, el entorno de la violencia es gradual y se acompaña de un sistema formal de comportamiento social configurado con rasgos patriarcales, de clase y de género, y con ayuda de valores que se socializan como herramientas de dominación que definen y soportan el imaginario colectivo patriarcal. Son estos valores dominantes los que se instalan en el espacio simbólico o material donde se desenvuelven las mujeres, dejándolas fuera del debate social y político (Galarza, Cobo \& Esquembre, 2016).

Ante esto, Pérez \& Radi (2018) sostienen que el desarrollo de la violencia motivada por el género es concebido por la legislación; sin embargo, se requiere una mirada más profunda, porque persiste una laguna hermenéutica, o sea, de la interpretación o traducción desde lo social que queda fuera del marco normativo.

La Organización de las Naciones Unidas (ONU, 1993) en la "Declaración sobre la Eliminación de la Violencia contra la Mujer" señala las características esta violencia, dándole el siguiente significado:

Se entiende todo acto de violencia basado en la pertenencia al sexo femenino que tenga o pueda tener como resultado un daño o sufrimiento físico, sexual o psicológico para la mujer, así como las amenazas de tales actos, la coacción o la privación arbitraria de la libertad, tanto si se producen en la vida pública como en la vida privada (p. 3).

El recorrido histórico de lucha por los derechos políticos y sociales por parte de las mujeres se ha caracterizado por la incesante confrontación para su reconocimiento (Puig, 2001), y se plantea que para la transformación de la realidad es necesario un enfoque integral que contemple las interrelaciones sistémicas entre el modelo económico y las prácticas sociales que sostienen la organización actual de satisfacción de necesidades sociales (Tapia, 2020).

En la actualidad, pese a que existe un enfoque normativo de las acciones que van en detrimento de los derechos de grupos sociales, el ámbito donde se desarrolla la violencia política en razón de género no es denunciado por miedo a ser revictimizada por parte de instituciones y profesionales encargados de prestar atención a la víctima, lo que crea el entorno propicio para que las manifestaciones de violencia sean invisibilizadas y normalizadas por la sociedad (Barrientos, 2019). 
Asimismo, el fenómeno de la feminización de los trabajos del hogar y de los cuidados prevalece en el entorno privado y se extiende a lo público. La violencia se propaga en ambos entornos, poniendo en desventaja exacerbada a las mujeres. Estas desventajas son expresadas desde la discriminación por género, la imposibilidad de desarrollo profesional y los límites que de esto resultan. Además de que los ataques hacia las mujeres por el solo hecho de ser mujeres tienen como raíz la descalificación y una discriminación sistemática hacia sus capacidades y posibilidades de hacer un buen trabajo o ganar una elección.

Es importante señalar que las mujeres en México han venido posicionando en la discusión pública los problemas comprendidos desde el pensamiento clásico como privados, del hogar o personales. Esto es romper con un proceso de violencia sistémica en un primer momento de forma simbólica, para después enarbolar logros sustantivos que hagan prevalecer el orden social de todas las personas.

Caminotti (2019) enmarca a la violencia política contra las mujeres con todo aquello que tenga como fin incidir negativamente en el ejercicio de poder o en el goce de derechos políticos, esto por razones de género. En la actualidad, estas manifestaciones de violencia política están también presentes en las redes sociales. Esta violencia política en razón de género corresponde a una de las estructuras sociales y no puede entenderse fuera de la estructura patriarcal.

En este sentido, el desarrollo de las luchas por la visibilidad pública y política de las mujeres en el espacio público es un rasgo característico de la lucha y resistencia que se gesta desde el feminismo. Constituyéndose las mujeres como movimiento social y como agentes sociales que se fusionan como grupo de presión, haciendo frente al androcentrismo de la política.

Es en el pacto patriarcal que se excluye a las mujeres del poder mediante las violencias simbólicas (Sandoval, 2019). En este sentido, este mismo autor señala que en México el entorno sociocultural legítima la exclusión de las mujeres por ser mujeres; es decir, se sostiene la violencia simbólica. $Y$ bajo este precepto se entorpecen y restringen los derechos políticos, lo que se traduce en violencia política en razón de género contra las mujeres.

\section{Método}

En este apartado se expone la aproximación del estudio mediante la clasificación del discurso de cada uno de los grupos analizados. Para el desarrollo de la metodología se trazó como pregunta de investigación: ¿cuáles son las barreras convencionales y no convencionales a las que se enfrentan las nuevoleonesas con doble jornada laboral para el ejercicio de la participación política?

El abordaje se realiza desde una perspectiva fenomenológica, para rescatar sus propias experiencias, a partir del entorno político y sociocultural.

Para lograr el objetivo del estudio se elaboró como instrumento de acopio de información una entrevista en profundidad, que se aplicó a doce mujeres en edades comprendidas entre 30 y 50 años, seleccionadas por medio de la técnica bola de nieve, en la que el contacto de la primer entrevistada sugirió el nombre y los datos de la siguiente, y que cumpliese con las características de la muestra. Se seleccionaron cuatro participantes para cada uno de los siguientes tres grupos de estudio; A) Servidoras Públicas; B) Integrantes de Organizaciones No Gubernamentales (ONG); C) Grupos de la Sociedad Civil Organizada (GSCO).

Para los fines del estudio se consideraron cuatro participantes para cada uno de los tres grupos, tomando en cuenta para su selección las siguientes características generales:

a) Ser mujeres

b) Tener una doble jornada laboral

c) Participar activamente en alguna organización política 
De manera específica para cada uno de los grupos:

Para el grupo A) Servidoras Públicas (SP): mujeres que participan en la política electoral postuladas a un puesto público, servidoras públicas municipales con cargos de dirección.

Para el grupo B) Organizaciones no Gubernamentales (ONG): mujeres dirigentes de organizaciones no gubernamentales con enfoque de participación en pro de los derechos humanos, migrantes y derechos políticos en transparencia gubernamental.

Para el grupo C) Grupos de la Sociedad Civil Organizada (GSCO): mujeres que participan desde plataformas no institucionales en pro de derechos de las niñas y los niños, feminismo y derechos sexuales y reproductivos y defensoras del territorio ante el despojo.

La incorporación de las cuatro participantes para cada grupo se especifica a continuación:

Al primer grupo corresponden: diputadas locales de la septuagésima quinta legislatura del Congreso de Nuevo León de los partidos PRI (1) y Movimiento Ciudadano (1) y dirección de la dependencia del Desarrollo Integral de la Familia con las siglas DIF en el municipio de la Zona Metropolitana de Monterrey (2).

$\mathrm{Al}$ segundo grupo lo forman: integrante de organización por los derechos de los migrantes (1), feminismo (2) y transparencia gubernamental.

Al tercer grupo lo integran: mujeres en pro de los derechos sexuales y reproductivos (1), agrupación feminista (1), agrupación vecinal en defensa del territorio (1) y derechos de las niñas y los niños (1).

Con la información aportada por las entrevistadas se consiguió generar en un primer abordaje una radiografía general del uso del lenguaje de las participantes entrevistadas, mediante la transportación de las transcripciones al software NVivo 11, para posteriormente analizar las respuestas de cada una y obtener la relación que guardan con el objetivo general del estudio.

Este mismo software cualitativo nos permitió conocer la frecuencia de palabras de todos los discursos, la clasificación de la información y agrupar los discursos.

Para la clasificación de los resultados se hizo primero una descripción general del discurso por cada uno de los tres grupos estudiados y, posteriormente, se unificaron los resultados que señalaban el objeto de estudio: identificar las principales barreras a las que se enfrentan las nuevoleonesas con doble jornada laboral en el ámbito de su participación política.

\section{Resultados discursivos de las nuevoleonesas con doble jornada laboral en el ejercicio de la partici- pación política}

A continuación, se exponen los resultados discursivos de las participantes mediante el uso de nubes de palabras generadas con el software NVivo 11. Se hace una clasificación por cada grupo de mujeres: A) Servidoras Públicas; B) Integrantes de Organizaciones No Gubernamentales (ONG); C) Grupos de la Sociedad Civil Organizada (GSCO), logrando identificar las principales expresiones y su relación con las experiencias en cuanto al contexto donde desarrollan su participación política.

\section{A) Servidoras públicas:}

A continuación, se ilustra por medio de una nube de palabras el discurso mayormente usado por el primer grupo de análisis, las cuales se presentan en la figura siguiente. 
Figura 1

Aportaciones de las Servidoras Públicas (SP)

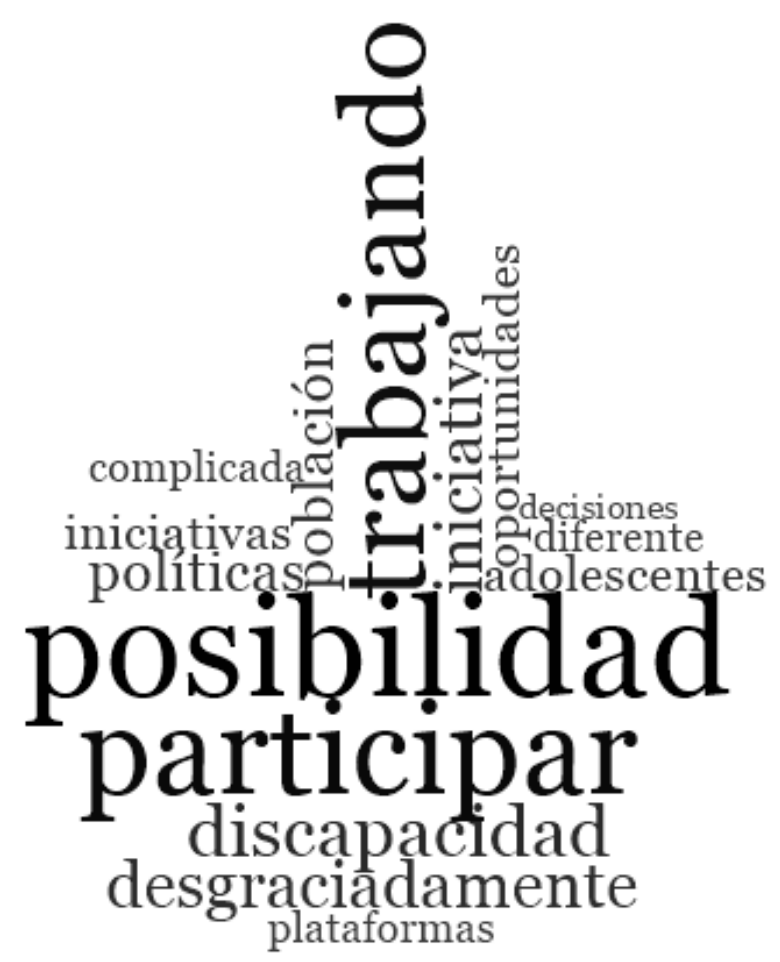

Nota. Palabras más destacadas por las servidoras públicas, elaboración propia con el software NVivo 11.

En el uso del lenguaje de las Servidoras Públicas se visualizó un contexto político-institucional, donde participan políticamente y desempeñan su actividad laboral remunerada. Las tres palabras con mayor frecuencia fueron: posibilidad, trabajando y participar, toman referencia con las posibilidades económicas, de redes, de logros profesionales, de toma decisiones, de flexibilidad de tiempo para eventos especiales con sus hijos e hijas y la posibilidad para emplear a una trabajadora del hogar. La posibilidad tiene relación íntima con privilegios desde el contexto económico, educativo y familiar.

Con relación a la palabra participar: acentúan la importancia de aportar mejoras a nivel social e incidir en cambios a nivel de desarrollo de las personas.
En cuanto a la palabra trabajando: consideran que se tuvo claridad con participar e incidir y trabajar en esferas políticas. Las participantes reflexionan en cuanto a la repercusión e impacto de sus decisiones encaminadas a impactar para ayudar a grupos vulnerables de la sociedad civil. Además, estiman que el estar trabajando permanentemente en pro de la sociedad es una responsabilidad y tienen en cuenta que como representantes de la sociedad civil es un gran compromiso que requiere permanencia para la transformación de la comunidad.

\section{B) Integrantes de Organizaciones No Guberna- mentales (ONG)}

En tanto, en el segundo grupo analizado se presentan como más recurrentes en su discurso las siguientes palabras:

\section{Figura 2}

Aportaciones de las Integrantes de Organizaciones No Gubernamentales

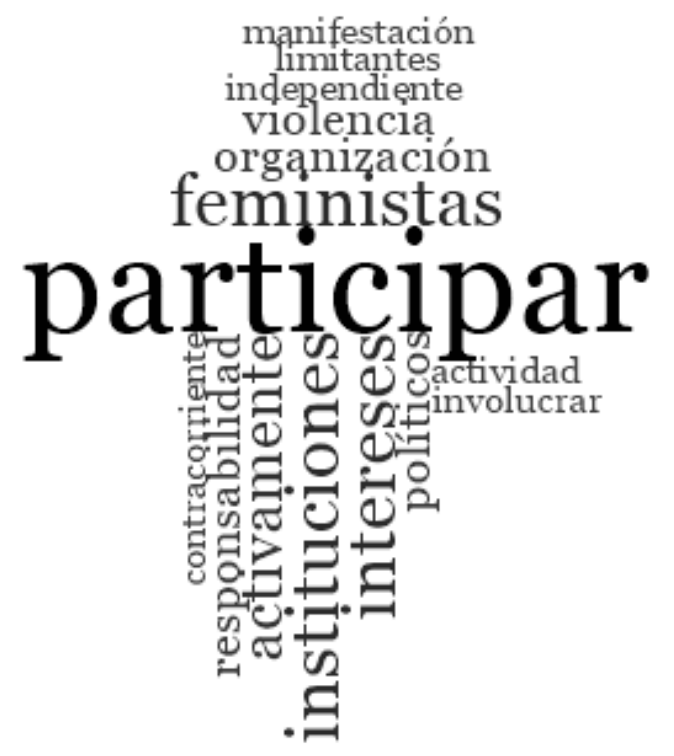

Nota. Palabras más destacadas por Integrantes de Organizaciones No Gubernamentales. Elaboración propia con el software NVivo 11. 
El grupo de las Organizaciones No Gubernamentales se integra por mujeres que trabajan con temas de Derechos Humanos, Transparencia Gubernamental y Feminismo. En cuanto a la primera palabra recurrente es participar: proyectan que las formas de participar han tenido que ser extensas entre manifestaciones multitudinarias y trabajo profesional y administrativo de las ONG.

Una de las palabras significativas de las mujeres partícipes de ONG es: instituciones. Admiten que el trabajo político de las organizaciones requiere una intensa relación con otras instituciones, además de que es la forma de continuar realizando el trabajo político para transformar la protección a los derechos humanos. Asumen que es de suma importancia que las mujeres estén representadas en todos los espacios de forma positiva, es decir, en la norma.

Referente a una de las tres palabras centrales del discurso es: intereses. Corresponde a las aspiraciones personales y punta de lanza para involucrarse en asuntos políticos. Asimismo, apunta a que los intereses creados por los poderes políticos son necesariamente cuestionables por los sectores organizados de la sociedad civil.

\section{C) Grupos de la Sociedad Civil Organizada (GSCO)}

El último grupo analizado es el que corresponde a mujeres participantes de Grupos de la Sociedad Civil Organizada (GSCO). Las actividades de este grupo se relacionan con aspectos de la salud sexual y reproductiva, organización que trabaja con niñas y niños desde la pedagogía, organización feminista. A continuación, se exponen las generalidades de su discurso.

\section{Figura 3}

Aportaciones de las Integrantes del Grupos de la Sociedad Civil Organizada (GSCO)

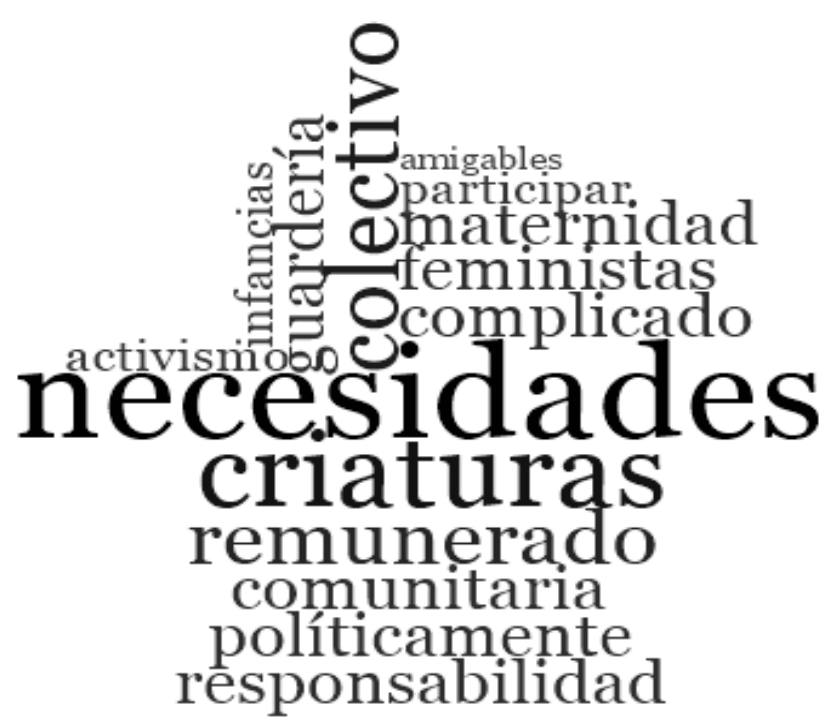

Nota. Palabras más destacadas por Integrantes del Grupos de la Sociedad Civil Organizada (GSCO), elaboración propia con el software NVivo 11.

La primera palabra recurrente se enfoca en las necesidades. El contexto de las necesidades de este grupo es propio de su problemática vida actual, la cual también responde a su interés por involucrarse en la solución de problemáticas sociales. Estas necesidades son de tipo: disposición de poco tiempo, dificultad para tejer redes y dificultades económicas.

La segunda palabra reiterada en su discurso es: criaturas. Es significativo en cuanto al campo de su activación política, dado que, en su mayoría, las mujeres de este grupo se ocupan de problemáticas que atañen afectaciones directas para el entorno de las familias y sus integrantes como lo son niñas y niños, denominados criaturas. Además de que para ellas la causa de su actividad política está íntimamente vinculada con la crianza y el desarrollo de sus hijas e hijos. 
La palabra que ocupa un tercer lugar es: colectivo. Es una palabra significativa en cuanto a la forma en la que realizan sus actividades políticas; creen indispensable la ejecución de su práctica de forma colectiva, tanto las decisiones como el ejercicio político.

\section{Barreras a las que se enfrentan las nuevoleonesas para el desarrollo de la participación política}

\section{A) Servidoras públicas:}

Dentro de este grupo de mujeres se afianza la posibilidad de participación política mediante los mecanismos formales y legales, como se muestra a continuación con una declaración:

“...los puestos [públicos] hace que se vea como incansable el poder participar o el poderestar ahí, ahorita las mesas de trabajo que hacemos son públicas, puede venir quienquiera, entonces es: cómo animarte en dónde te interesa participar, ve, lo que más urgeson manos en todos los aspectos". (Entrevistada 1).

La maternidad se expone como una limitante para participar plenamente en la política, tal como se describe en la teoría presentada con anterioridad. Además, se relaciona el logro de puestos directivos [o de poder] con la soltería y con no tener responsabilidades familiares. Una de las participantes al respecto comenta:

"las barreras nos las ponemos nosotras desde el momento en el que tenemos familia, sin duda yo sí veo esa [barrera]... desde que yo trabajé en la Gamesa y las directoras mujeresno tenían familia y muchas veces eran solteras". (Entrevistada 2)

También, suman una barrera en la participación política electoral, enfocada en: la distribución inequitativa entre hombres y mujeres de los distritos ganables para un partido político. Asumiendo que las candidaturas a los distritos con pocas posibilidades de triunfo son entregadas a las mujeres militantes y no a los hombres. Una de las declaraciones que nos comparte una entrevistada se expone a continuación:

“muchas personas [candidatas] me decían que sí lo vivieron de esa manera [inequidad enel reparto de candidaturas], como diciendo: «no, es que ya trabajamos mucho esteterreno, vas a ver que va a ser diferente el resultado" [expresión del partidopolítico que las postula] y por supuesto que llegabas [a la circunscripción del distrito] y tedecían: «a ver, aquí nadie se ha parado» [risas], pues el comité municipal probablementeno había estado ni siquiera activo". (Entrevistada 3)

Por último, mencionan que hay poca sororidad a la hora de apoyar iniciativas entre mujeres legisladoras representantes de algún distrito. Una de las diputadas comparte:

"falta mucho el tema de la sororidad, o sea, de mujeres apoyarnos a las mujeres porqueseguimos en un país... y yo lo veo aquí en la legislatura, en donde a veces o los celos o lasenvidias nos ganan para poder hacer algo grande, ¿cuál? [legisladora], el que sea, grandeposiblemente con sus iniciativas, o en este caso, ella sea grande con sus iniciativas... Estova a beneficiar a todo Nuevo León”. (Entrevistada 4)

\section{B) Integrantes de Organizaciones No Guberna- mentales (ONG)}

Respecto de las barreras identificadas por las mujeres integrantes de ONG, concuerdan en que el impacto de la inseguridad tiene una relación directa con la posibilidad de continuar involucrándose en la política. Una de las participantes menciona: 
“siento yo que vamos para atrás, pero yo parto de una base, mientras la seguridad no se vuelva [regrese] un 2010 [situaciones de: desapariciones,secuestros, enfrentamientos entre cárteles, etc.] que fue lo vivimos, yo ahí suelto el arpa y ahí paramos de participar”. (Entrevistada 5)

En el mismo sentido, otra participante de una ONG sostiene que las amenazas contra el involucramiento político es una forma de exponer su seguridad y que acota las posibilidades de dedicarse a la política. Con relación a lo anterior, una participante comparte:

“yo tenía una aspiración de dedicarme a la política, lo que no te imaginas, cuando tú temetes a un organismo de la sociedad civil y más en materia migratoria, es que estásexpuesta a cuestiones de amenazas, cuestiones de que te estén presionando... no elgobierno, esperaría que no me hiciera nada, no es lo mismo que te amenace fulanito a quete amenace el gobierno, porque contra el gobierno no vas a poder". (Entrevistada 6)

La presencia de violencia es un aspecto limitante para las mujeres integrantes de las ONG. En este sentido, una de las participantes expone que la represión policiaca produce limitantes para implicarse en asuntos de índole social y organizativo. A continuación, una participante menciona:

"Hace poco... en el 28S [Ley: interrupción voluntaria del embarazo/aborto legal enArgentina con extensión en Latinoamérica], pues tuvimos represión de la policía, obviamente esa represión te limita de alguna manera a que sigas... participando. Hay quienes tienen miedo de volver a salir a la calle, o por su seguridad se cubren el rostro, incluso por el "qué dirán", todos esos son limitantes para que podamos participar deforma libre". (Entrevistada 7)

También identifica: la jerarquización de la política por género, tomando en cuenta más a los hombres para responsabilidades mayores. Señalan una asimetría en el ejercicio de equidad con los hombres a la hora de responsabilidades políticas. Distinguen esta práctica como violenta. Una integrante de ONG expresa lo siguiente:

"la jerarquización de las responsabilidades es una forma que vivimos y que esviolencia... debemos entender que todos somos parejos y somos iguales". (Entrevistada 7)

\section{C) Grupos de la Sociedad Civil Organizada (GSCO)}

En el último grupo estudiado, el cual está conformado por mujeres que participan en colectivos no institucionales y/o legalmente constituidos, se identifica que: hay una barrera expresa con el establecimiento de la sociedad patriarcal. Dado que esta posición antepone las necesidades e intereses propios de la mujer por el cuidado y la dedicación a las hijas e hijos, esta relegación de sí misma se da a partir de lo que los roles tradicionales imponen al ser madre o mujer. Es importante señalar que se distingue esta práctica como una agresión pasiva, es decir, violentar mediante el entorpecer o afectar las tareas o los proyectos de las mujeres. Una de las participantes expresa:

"desde el hecho que llevamos una sociedad patriarcal pues implica muchos discursos,como primero, ten la casa limpia y luego hazte cargo de tus hijos o los traes ahí [en elespacio público de participación] cuando hace calor o los traes cuando hace mucho frío oya es muy noche y se desvelan, y no es justo que antepongas las necesidades a las de lacriatura, o sea, se espera que una mujer se vuelque hacia sus hijas o a sus hijos, que dejede lado todas sus necesidad para atender esas, entonces... este discurso es pasivoagresivo". (Entrevistada 8)

Una de las barreras identificadas para este grupo de mujeres tiene que ver con limitantes materiales/ económicas y de movilidad. Esto en relación a que 
la práctica política de las mujeres del grupo la manifiestan en el espacio público, en colonias y centros vecinales, por tanto aprecian que la movilidad forma una limitante para la plena participación política. A continuación, una participante explica:

“están todos estos obstáculos como el varo [dinero] o la poca facilidad de moverte... lamovilidad está muy mal porque los camiones siempre van muy llenos, porque no haysuficientes unidades, porque las rutas no te llevan a ninguna parte, o sea, te dejan bienlejos de donde realmente quieres estar, son todas [las rutas de transporte], donde estés".(Entrevistada 9)

Un enfoque significativo que exponen las mujeres de grupos de la sociedad civil organizada es relacionado con las acotadas posibilidades para acceder a guarderías. También identifican la imposición de la maternidad y dedicación exclusiva al cuidado de las hijas. Además de la reducción de seguridad social para mujeres con empleos informales, que no pueden acceder a un servicio de guardería privada. Sin considerar políticas que creen las condiciones necesarias para que las mujeres se involucren en los asuntos de la política. Una de las participantes comparte su experiencia:

“...Esas guarderías De Sedesol o de bienestar solo son para las madres que tienen trabajoo que estén buscando trabajo. Entonces si tú no tienes forma de comprobar que tienes unempleo no puedes acceder a un servicio, que es como el más económico, puedes accedera otros servicios privados, pagar una guardería... si yo no trabajo o no tengo un trabajoremunerado, o tengo $\mathrm{mi}$ propio negocio vendo sabalitos, ¡qué sé yo!, pero quiero untiempo sin mi hija porque quiero escribir un libro o quiero hacer un programa, lo que sea,no puedo llevarla a una guardería comunitaria vecinal o a una guardería del gobiernoporque no tengo un trabajo. El gobierno está diciendo que la única razón buena para dejara tus hijos es que estés trabajando, o sea no hay otra razón por la cual tú deberías de haceruna cosa que no sea cuidar a tu hijo. Es un mensaje muy fuerte." (Entrevistada 10)

La presencia de estereotipos estéticos impuestos para las mujeres, además de normas socialmente aceptadas mediante la imposición de los rasgos aceptados como femeninos, revelan barreras para que las mujeres participen en la política. Estos estereotipos enaltecen características físicas de las mujeres antes que a su preparación académica y/o logros profesionales. Ante esto, una participante refiere su experiencia:

"Como mujer, tienes que cumplir con ciertas normas sociales o institucionales deestética... para que puedas estar [participando], el simple hecho de que físicamente tienesque cumplir con estereotipos para ser aceptada. A pesar de que puedas tener experiencia,grados académicos; realmente lo que se toma en cuenta son otros aspectos... sobre todovisibles... de cumplir con estereotipos que marcan la sociedad y las instituciones.Entonces, eso es también para mí una barrera”. (Entrevistada 11)

\section{Conclusiones}

Las limitantes y los condicionamientos que rodean a las mujeres que participan en asuntos políticos están vinculados a la sociedad patriarcal y se combina con características de origen sistémico. A causa de las desigualdades materiales se crean o no oportunidades de desarrollo para las mujeres, ya sea dentro de entornos profesionales, familiares y políticos.

Además, el establecimiento de roles de género en la sociedad se identifica con una singularidad: la imposición de convertir a las mujeres en sostenes de la producción y el sostenimiento de la vida de las otras personas, atribuyendo incluso estereotipos limitantes que impactan en el desarrollo integral de las mujeres. En este sentido, se construye una sociedad patriarcal dentro y fuera del hogar, que implica que 
las mujeres sean sojuzgadas al no cumplir con los roles establecidos para este tipo de sociedad: dedicación al trabajo del hogar y los cuidados de sus integrantes, así como la maternidad.

El primer acercamiento del estudio, mediante la identificación discursiva de las participantes, permitió explorar la manera en que el contexto de vida y el desarrollo de las mujeres en Nuevo León tiene relación con el interés y el enfoque que le dan a sus prácticas en la política. Se encuentra que mientras más tradicional sea la participación política, como la política electoral e instituciones establecidas legalmente, se reconoce un ambiente de paridad entre hombres y mujeres para participar. Además, se rescata la importancia de ocupar el espacio de la política y de las decisiones por parte de las mujeres.

Mientras que la participación de mujeres en grupos o colectivos no constituidos enfrenta obstáculos en la seguridad social y el ambiente de violencias simbólicas y materiales, no dejan de igual manera de estar sujetas al sometimiento de estereotipos de género establecidos históricamente.

Las barreras que identifican las mujeres del primer grupo estudiado (A) Servidoras Públicas se relacionan con la maternidad y el estado civil; encuentran las mujeres que al convertirse en madres o tener responsabilidades de pareja y familiares impiden logros profesionales y políticos significativos. Sin embargo, también manifiestan que hay mayores oportunidades para las mujeres gracias a que hay plataformas políticas para participar políticamente.

Señalan que la masculinización de la política se da dentro de los partidos y que hay una distribución inequitativa de las candidaturas, que en ocasiones se privilegia a los hombres para otorgarles mayores oportunidades de triunfo.

El segundo grupo, (B) Organizaciones no Gubernamentales, lidera el discurso de manera institucional e incluso pugnan por que la presencia de las mujeres se de en los ámbitos públicos y políticos para cambiar las condiciones sociales que las ponen en desventaja. En este grupo se identifican barreras políticas relacionadas con las consecuencias de la violencia suscitada en todo México, incluso, su interés puede ser limitado de forma racionalizada: asumiendo que la violencia e inseguridad puede ser causante para desistir en seguir involucrándose en asuntos públicos y políticos.

Además, de que las represiones policiacas experimentadas al ejercer sus formas de participación política crean un entorno de miedo, de tal forma que conducen al abandono de hacerse visibles en el espacio público. Las formas de violencia en razón de género la experimentan también con la jerarquización masculinizada de las responsabilidades políticas, asumiendo que las causas de mayor importancia en las organizaciones se les atribuye a los hombres.

Las mujeres en el grupo C), Grupos de la Sociedad Civil Organizada, enfatizan que los estereotipos que imponen un tipo de feminidad destinado a fomentar la sociedad patriarcal llevan a normas sociales que acarrean barreras para el desenvolvimiento integral de las mujeres. Refieren que una sociedad patriarcal construye un orden de desigualdades materiales. Esta sociedad no fomenta las posibilidades de logros y políticas que contrarresten los obstáculos por el olvido de políticas sustantivas para el desenvolvimiento de las mujeres en la política. Por el contrario, se afianzan estereotipos que resaltan cualidades físicas de las mujeres frente a los logros profesionales.

Por tanto, las barreras frente a la política de las mujeres nuevoleonesas con doble jornada laboral toman una perspectiva particular e incluso entre las integrantes de cada uno de los grupos. Se puede ubicar el entorno de las desigualdades como simbólicas e históricas y materiales. Un rasgo importante para desarrollar como futura línea de investigación es la relación de la clase social, así como los ambientes socializadores de las mujeres vinculados a la elección de involucrarse en la política. 


\section{Referencias}

Álvarez Rudín, M. (2011). Movimientos Sociales y participación política: El movimiento contra El TlC en la campaña del referéndum 2007 en Costa Rica. Anuario de Estudios Centroamericanos, 37, 201-230. http://www.jstor. org/stable/41306439

Barrientos Zepeda, E. (2019). La Cosmovisión indígena y la mujer en la política. Reflexiones del caso mexicano. Ius Comitialis, 2(3), 222-247. https://doi.org/10.36677/iuscomitialis. v2i3.12173

Benhabib, S. (1997). The Embattled Public Sphere: Hannah Arendt, Juergen Habermas and Beyond. Theoria: A Journal of Social and Political Theory, (90), 1-24. http://www.jstor.org/ stable/41802076

Butler, J. (2007). El género en disputa: el feminismo $y$ la subversión de la identidad. Paidós. http:// www.lauragonzalez.com/TC/El_genero_en_ disputa_Buttler.pdf

Caminotti, M. (2019). Cuando hacer política te cuesta la vida. Estrategias contra la violencia política hacia las mujeres en América Latina, de Flavia Freidenberg y Gabriela del Valle Pérez (Eds.). Perfiles latinoamericanos, 27(54).

Cerva Cerna, D. (2014). Participación política y violencia de género en México. Revista Mexicana de Ciencias Politicas y Sociales, 59(222), 117-139. https://www.redalyc.org/articulo. oa?id=421/42131768005

Código Federal de Instituciones y Procedimientos Electorales, IFE, México, 2008. http://www. diputados.gob.mx/LeyesBiblio/abro/cofipe/ COFIPE_abro_14ene08.pdf

Dávila Jácome, P. M. (2018). Redes de cuidado y su relación con la sobre carga de trabajo de las madres dentro de los mercados municipales de Quito, año 2017 [Tesis de Licenciatura, Pontificia Universidad Católica del Ecuador]. http://repositorio.puce.edu.ec/handle/ $22000 / 14646$ ? show=full
Donoso, A. \& Valdés, T. (2007). Participación politica de las mujeres en América Latina. Informe regional. Asociación Latinoamericana de Organizaciones de Promoción para el Desarrollo AC. http://www.lasociedadcivil.org/wp-content/ uploads/2014/11/alop_informe_regional_00_ pp_mujeres_al_txt_completo11.pdf

Diario Oficial de la Federación, "Decreto por el que se expide la Ley General de Partidos Políticos", Estados Unidos Mexicanos, 23 de mayo de 2014, http://dof.gob.mx/nota_detalle.php?codigo $=5345955 \&$ fecha $=23 / 05 / 2014$.

Diario Oficial de la Federación, "Decreto por el que se expide la Ley General de Partidos Políticos", Estados Unidos Mexicanos, 23 de mayo de 2014, http://dof.gob.mx/nota_detalle.php?codigo $=5345955 \&$ fecha $=23 / 05 / 2014$.

Pedrero Nieto, M. (2018). El trabajo y su medición. Mis tiempos. Antología de estudio sobre trabajo y género. México: Universidad Nacional Autónoma de México, Centro Regional de Investigaciones Multidisciplinarias / Miguel Ángel Porrúa, 847 p. [Revisión del libro El trabajo y su medición. Mis tiempos. Antología de estudio sobre trabajo y género, por M. Pedrero]. Estudios Demográficos y Urbanos, 35(3), 839-848. http://dx.doi.org/10.24201/edu.v35i3.2040

Federici, S. (2013). Revolución en punto cero. Trabajo doméstico, reproducción y luchas feministas. Traficantes de Sueños. https://www.traficantes.net/sites/default/files/pdfs/map36_ federici.pdf

FernándezHasan,A. (2007). Desigualdad de género. La segregación de las mujeres en la estructura ocupacional. La ventana. Revista de estudios de género, 3(25), 140-167. http://www. scielo.org.mx/scielo.php?script=sci_arttext $\&$ pid=S1405-94362007000100140\&lng= es\&tlng=es

Galarza Fernández, E., Cobo Bedía, R. \& Esquembre Cerdá, M. (2016). Medios y violencia simbólica contra las mujeres. Revista Latina de Comunicación Social, 71, 818-832. https:// doi.org/10.4185/RLCS-2016-1122 
Godoy, L. \& Mladinic, A. (2009). Estereotipos $\mathrm{y}$ roles de género en la evaluación laboral y personal de hombres y mujeres en cargos de dirección. Psykhe (Santiago), 18(2), 51-64. https://scielo.conicyt.cl/pdf/psykhe/v18n2/ art04.pdf

Ibarra, V. E. (2018). Brecha de género y feminización de la pobreza en América Latina: una aproximación desde el informe de ONU Mujeres 2017 y la perspectiva de Federici. Etcétera. Revista del Area de Ciencias Sociales del CIFFyH, 2. https://revistas.unc.edu.ar/index. php/etcetera/article/view/22063/22587

La Parra, D. (2001). Contribución de las mujeres y los hogares más pobres a la producción de cuidados de salud informales. Gaceta Sanitaria, 15(6), 498-505. https://doi.org/10.1016/ S0213-9111(01)71612-3

Lena Krook, M. \& Restrepo Sanín, J. (2016). Género y violencia política en América latina. Conceptos, debates y soluciones. Política y Gobierno, 23(1), 127-162. https://www. redalyc.org/pdf/603/60343614006.pdf

Ley General de Instituciones y Procedimientos Electorales (LEGIPE). 23 de mayo 2014. http:// www.diputados.gob.mx/LeyesBiblio/pdf/ LGIPE_130420.pdf

Ley General de Partidos Políticos (LGPP). 23 de mayo 2014. http://www.diputados.gob.mx/ LeyesBiblio/pdf/LGPP_130420.pdf

Ocampo Salazar, J. F. (2016). Trabajo doméstico: familia, roles de género y división sexual del trabajo. Una experiencia con los estudiantes de ciclo cinco de colegios oficiales de la localidad de Usaquén. En E. N. Agudelo y D. A. Restrepo (Coords.), Diálogos en perspectivas de género: reflexiones sobre la escuela, diversidad y diferencia (pp. 258-272). Universidad Pedagógica Nacional. http://hdl.handle.net/ $20.500 .12209 / 11456$

Organización de las Naciones Unidas (ONU), Asamblea General. (Diciembre, 1993). Declaración sobre la eliminación de la violencia contra la mujer: Resolución de la Asamblea General
48/104 del 20 de diciembre de 1993. https:// www.refworld.org.es/docid/50ac921e2.html

Ortega, J. (2019). Trabajos de(s)cuidados: reformas neoliberales, feminización y precarización de las condiciones de trabajo en enfermería. Athenea Digital. Revista de pensamiento e investigación social, 19(3), 1-26. https:// doi.org/10.5565/rev/athenea.2333

Peris Vidal, M. (2013). La despolitización de la violencia de género a través de la terminología. Asparkía. Investigació Feminista, (24), 176-194. https://www.e-revistes.uji.es/index. php/asparkia/article/view/1169

Pérez, M. \& Radi, B. (2018). El concepto de 'violencia de género' como espejismo hermenéutico. Igualdad, autonomía personal y derechos sociales, 8, 69-88. https://www.aacademica.org/moira. perez/49

Pérez, M. (2019). Violencia epistémica: reflexiones entre lo invisible y lo ignorable. El lugar sin límites. Revista de Estudiosy Politicas de Género, 1(1), 81-98. http://revistas.untref.edu.ar/ index.php/ellugar/article/view/288/267

Puig, A. I. M. (2001). Los derechos políticos de las mujeres: evolución y retos pendientes. Cuadernos Constitucionales de la Cátedra Fadrique Furió Ceriol, (36-37), 195-214. https://dialnet.unirioja.es/servlet/articulo?codigo $=623913$

Rodríguez Enríquez, C. M. (2015). Economía feminista y economía del cuidado: Aportes conceptuales para el estudio de la desigualdad. Nueva sociedad, 256, 30-44. https://biblat.unam.mx/ hevila/Nuevasociedad/2015/no256/3.pdf

Silva Marińos, L. (2020). ¿ ‘Tabajos esenciales pero no reconocidos? Una mirada desde la economía popular frente a la pandemia del COVID-19. Trabajo y sociedad, 35(21), 27-49. https://ri. conicet.gov.ar/handle/11336/109680

Sandoval Vázquez, F. R. (2019). Democracia feminista y violencia política en México. Inventio, la génesis de la cultura universitaria en Morelos, 15(36), 21-29. http://inventio.uaem.mx/ index.php/inventio/article/view/551/1284 
Tapia Marchina, S. (2020). Una lente feminista de la desigualdad económica. Debate Feminista, 60, 24-47.10.22201/cieg.2594066xe.2020.60.02 Vázquez García, V., Cárcamo Toalá, N. J. \& Hernández Martínez, N. (2012). Entre el cargo, la maternidad y la doble jornada: presidentas municipales de Oaxaca. Perfiles latinoamericanos, 20(39), 31-57. http://www. scielo.org.mx/scielo.php?script $=$ sci_arttext\&pid=S0188-76532012000100002

Vázquez Correa, L. \& Patińo Fierro, M. P. (2020) Violencia política contra las mujeres y paridad de género: de la presencia en el poder a la transformación de la política. Cuaderno de investigación, 67, Instituto Belisario Domínguez, SenadodelaRepública.http://bibliodigitalibd. senado.gob.mx/handle/123456789/4864

Zabludovsky, G. (2007). Las mujeres en México: trabajo, educación superior y esferas de poder. Politica y cultura, 28, 9-41. http://www. scielo.org. $\mathrm{mx} / \mathrm{scielo}$.php?script=sci_arttext\&pid=S0188-77422007000200002\&ln$\mathrm{g}=\mathrm{es} \& \mathrm{t} \operatorname{lng}=\mathrm{es}$ 